\title{
Testing for Asymmetric Nonlinear Short- and Long-Run Relationships between Bitcoin, Aggregate Commodity and Gold Prices
}

\author{
Elie Bouri \\ USEK Business School, Holy Spirit University of Kaslik (USEK), POB 446 Jounieh, \\ Lebanon. Email: eliebouri@usek.edu.lb \\ Rangan Gupta \\ Department of Economics, University of Pretoria, Pretoria, South Africa. Email: \\ rangan.gupta@up.ac.za.
}

Amine Lahiani

LEO, University of Orleans, Orléans - France, Montpellier Business School, Montpellier France. Email: amine.lahiani@univ-orleans.fr

Muhammad Shahbaz

Montpellier Business School, Montpellier - France. Email: muhdshahbaz77@gmail.com

\begin{abstract}
Unlike prior studies, this study examines the nonlinear, asymmetric and quantile effects of aggregate commodity index and gold prices on the price of Bitcoin. Using daily data from July 17, 2010 to February 2, 2017, we employed several advanced autoregressive distributed lag (ARDL) models. The nonlinear ARDL approach was applied to uncover short- and longrun asymmetries, whereas the quantile ARDL was applied to account for a second type of asymmetry, known as the distributional asymmetry according to the position of a dependent variable within its own distribution. Moreover, we extended the nonlinear ARDL to a quantile framework, leading to a richer new model, which allows testing for distributional asymmetry while accounting for short- and long-run asymmetries. Overall, our results indicate the possibility to predict Bitcoin price movements based on price information from the aggregate commodity index and gold prices. Importantly, we report the nuanced result that most often the relations between bitcoin and aggregate commodity, on the one hand, and between bitcoin and gold, on the other, are asymmetric, nonlinear, and quantiles-dependent, suggesting the need to apply non-standard cointegration models to uncover the complexity and hidden relations between Bitcoin and asset classes.
\end{abstract}

Keywords: Cointegration, Asymmetry, Nonlinearity, Quantile Dependence, Bitcoin, Commodity, Gold

JEL Classification: C12, G15, Q02 


\section{Introduction}

Derived from mathematical cryptography, and based on peer-to-peer networking, Bitcoin was the first, and is the most popular digital currency to come into existence. Unlike conventional sovereign currencies, it operates without the need for any intermediary, and is not a liability on any balance sheet. Its decentralized and pseudo-anonymous attributes make it appealing to users, thanks to two key innovations: The first is the public-distributed ledger, called 'blockchain' where all Bitcoin transactions are digitally recorded. The second is the consensus mechanism, called 'proof of work', which is used to solve the double-spend problem.

After being proposed by Nakomoto (2008) in a paper entitled "Bitcoin: A Peer-to-Peer Electronic Cash System", Bitcoin was implemented on January 3, 2009 as an alternative payment system independent of any central authorities or central banks. However, Bitcoin emerged later as an investment asset (Polasik et al., 2015), given the tradability of its unit of value. Notably, the accessibility aspect of Bitcoin as an investment has been enhanced by the inception of trading platforms where Bitcoin is bought and sold using traditional currencies. Accordingly, many market participants have arisen to profit from movements in the Bitcoin price. In a seven-year period, the Bitcoin price has increased exponentially from less than one USD in April 2011 to 1,347.96 USD at the end of April 2017.

Scholars were first interested in the technical, safety, ethical, and legal aspects of Bitcoin; then the economics and financial aspects became the subject of interest. Fry and Cheah (2016) provide evidence on the Bubble in the Bitcoin market, and similar results are reported by Cheung et al. (2015), who indicate that Bitcoin has no fundamental value, but instead a mostly speculative value. Quite similar results are shown by Baek and Elbeck (2015), who indicate that Bitcoin is 26 times more volatile than the S\&P 500 Index and that Bitcoin returns are not influenced by fundamental economic factors, but rather by buyers and sellers. Given that Bitcoin does not have identifiable cash flows, some studies have raised the difficulty in determining its fundamental value and suggest instead empirical models to identify the determinants of the Bitcoin price. For instance, Bouoiyour and Selmi (2015) show that in the short term, the Bitcoin price is appositively affected by investors' attraction and the exchangetrade ratio. Kristoufek (2015) employed a wavelet coherence approach and indicates that the Bitcoin price is not affected by economic and financial variables. Further examination of price formation in the Bitcoin market points toward the importance of market forces, Bitcoin's attractiveness, and the insignificance of macro-financial developments (Ciaian et al., 2016). However, Li and Wang (2017) indicate that, in the long term, Bitcoin's price is more sensitive 
to economic fundamentals and less sensitive to technological factors. Yelowitz and Wilson (2015) reveal that computer programming enthusiasts and illegal activity have driven interest in Bitcoin but found no support for political and investment motives. Ober et al. (2013) stress the important role of user anonymity in the Bitcoin market.

The usefulness of Bitcoin as an investment, and its potential hedging and safe-haven roles have been the subject of several studies. Baur et al. (2015) show that Bitcoin is an investment, and highlight its role as a useful diversifier (i.e. uncorrelated with traditional assets). Brière et al. (2015) used weekly data from 2010 to 2013 and highlight the low correlation of Bitcoin with both traditional assets (worldwide stocks, bonds, hard currencies) and alternative investments (commodities, hedge funds, real estate), and point toward the significant diversification benefits of Bitcoin, despite its extremely high average return and volatility. They also show that adding a small proportion of bitcoins (3\%) can enhance the risk-return trade-off of well-diversified portfolios. Dyhrberg (2016b) indicates that Bitcoin is a hedge for UK currency and equities. Ji et al. (2017) argue that Bitcoin is isolated from the global financial system, suggesting a valuable role in portfolio diversification. Bouri et al. (2017c) show that Bitcoin is an effective diversifier for major world equities, bonds, oil, gold, the general commodity index, and the US dollar index.

Bitcoin is often seen as a basic form of movement against capitalism, free trade, and globalization (Cohen, 2016), and is part of an alternative 'peer-to-peer' economy (Bouri et al. $2017 \mathrm{c}$ ). That Bitcoin emerged in 2009, the year that followed the global financial crisis, during which an environment of weak trust in the global financial system had spread, points toward the potential role of Bitcoin not only as an alternative to cash (Evans-Pughe, 2012), but also as the panacea to replace financial institutions (Kerner, 2014). In this sense, Bitcoin has been appreciated by practitioners and users, especially during the numerous financial and economic crises that have coincided with its short history. Notably, during the European sovereign debt crisis of 2010-2013 and the Cypriot banking crisis of 2012-2013, Bitcoin was seen as an alternative currency to those of conventional economies and a shelter from sovereign and systematic risk (Bouri et al. 2017b). Luther and Salter (2017) show that interest in Bitcoin amplified considerably after Cyprus announced it would accept a bailout. Bitcoin has been seen by many as an alternative to traditional stores of value, such as gold, and has been named the digital gold (Popper, 2015). Several articles refer to a flight from conventional currencies to Bitcoin, a feature that is often associated with gold. Dyhrberg (2016a) locates the hedging capability of Bitcoin somewhere near to that of gold. However, there is no consensus among scholars about whether Bitcoin is a currency or commodity, or 
both (Lo and Wang, 2014). Yermack argues that Bitcoin does not fulfil the functions of money, whereas Polasik et al. (2015) show that it acts as a medium of exchange. Recently, Blau (2017) showed that Bitcoin is a currency rather than a speculative investment. Importantly, Bitcoin combines features from commodities (i.e. gold) and sovereign currencies, and thus can be considered as synthetic commodity money (Selgin, 2015). Baur et al. (2015) argue that Bitcoin is a hybrid between precious metals and conventional currencies. Interestingly, in April 2017 the Bitcoin price for the first time exceeded the price of an ounce of gold, and numerous press articles emerged comparing Bitcoin and the yellow metal.

While gold differs from Bitcoin in several aspects, such as history, tangibility, intrinsic value, low volatility, consumption or use in the production process, it shares with Bitcoin several important characteristics. First, both gold and Bitcoin are regulated as commodities, especially in the US, where Bitcoin has recently been classified as a commodity by the Commodity Futures Trading Commission (CFTC). Second, Bitcoin has non-political and (virtual) commodity attributes that make it quite similar to gold. As such, the absence of a central authority to control and adjust Bitcoin mining and transactions makes Bitcoin independent of inflation (Richardson, 2014; Baur et al., 2015), an important feature commonly associated with gold. Third, and like gold, Bitcoin is produced in a process called "mining" and also has limited supply and supply growth, as dictated by Bitcoin protocol. The latter ensures that a maximum of 21 million coins can be mined. In countries like China and Russia, Bitcoin represents a solution for taking cash out of the country, while circumventing strict regulations on capital flows. Furthermore, the recent scrutiny by the Chinese government over the physical gold market has made Bitcoin an ideal alternative to gold.

Numerous studies have examined the relations between Bitcoin and asset classes such as stocks, bonds, and currencies. However, it remains unclear what relation exists between Bitcoin and commodities in general, and between Bitcoin and gold in particular, especially in terms of asymmetry, non-linearity, and quantiles variability. To address this literature gap, in this study we examined the nonlinear, asymmetric, and quantile effect of aggregate commodity and gold prices on the Bitcoin price, using a combination of autoregressive distributed lag (ADRL) models, namely the nonlinear ARDL (NARDL) model of Shin et al. (2011) and the quantile ARDL (QARDL) model of Cho et al. (2015). We also applied a combination of these two models, called the Quantile Nonlinear Autoregressive Distributed Lag (QNARDL) model. In addition to the novel contribution presented in this paper, by allowing for asymmetric behavior between the examined variables (i.e. by applying the hidden cointegration analysis), we offer another novelty. This entails accounting for a second 
type of asymmetry, known as distributional asymmetry according to the position of the dependent variable within its own distribution. Such a combination of nuanced integration approaches has not been used in the literature to date. The proposed QNARDL model allows testing for distributional asymmetry, while accounting for long- and short-run asymmetries. Although relations among Bitcoin and several economic and financial variables, such as the general commodity index and the gold price, have been examined in prior studies, the potential presence of asymmetric, non-linear effects, and quantile variability in these relations has been ignored. For example, Bouri et al. (2017c) report evidence of the ability of Bitcoin to diversify movements in conventional assets, commodities, and energy commodities. Ji et al. (2017), who used a directed acyclic graph approach and focused on the integration of the Bitcoin market within the global financial system, reveal a very weak relation between Bitcoin and commodity markets, including the gold market. Bouri et al. $(2017 \mathrm{~b}, \mathrm{c})$ relied on quantile dummies within a dynamic conditional correlation framework to assess the safe haven property of Bitcoin against commodities, but the authors do not account for the cointegration relationship across the quantiles. Although Bouri et al. (2017a) employed a quantile-based approach, they focused only on the relation between Bitcoin and global uncertainty. Balcilar et al. (2017) employed a nonparametric causality-in-quantile test to unveil the causal relationship between trading volume and Bitcoin returns and volatility. They highlight the importance of considering a quantile nonlinear approach to detect causality in different quantiles.

There are few studies that used an ARDL model and focused on price discovery in the Bitcoin market (Bouoiyour and Selmi, 2015; Ciaian et al., 2016; Li and Wang, 2017), but conflicting findings have emerged. Importantly, these studies do not account for asymmetric and nonlinear effects, and assume that the examined relation is homogenous across the different quantiles. Ciaian et al. (2016) show that the Bitcoin price is not sensitive to macro-financial developments in the long run. Li and Wang (2017) found a significant relation between the Bitcoin price and changes in economic fundamentals, both in the short and long runs. Bouoiyour and Selmi (2015) failed to detect any significant relation between the Bitcoin and gold markets.

Recently, there have been some interesting developments in econometric modelling based on the ADRL framework, which have led to the emergence of the NARDL model (Shin et al. 2011) and the QARDL model (Cho et al. 2015). When applied to the relation between Bitcoin and aggregate commodity (gold) prices, these models allow us to provide a clear view of the relation, which is useful to market participants. The latter are keen to understand non- 
linearity, asymmetry, and variability across the quantiles, which ultimately would help them generate better trading and investment strategies. In our empirical analysis, we used the aggregate commodity index and gold prices separately as the main dependent variables, and Bitcoin as the dependent variable. We also included several explanatory variables stemming from the literature. Accordingly, our analysis covers a large number of assets that includes not only conventional assets such as equities, bonds, and currencies, but also commodities. In examining commodities, and in addition to a general commodity index, we focused on gold, as many press and scholarly articles refer to Bitcoin as a "digital" commodity, or "digital" gold. We also focused on the dollar index, given the wide acceptance and use of Bitcoin as a digital currency, although no consensus exists on whether Bitcoin is a currency, commodity, or both (a hybrid asset). Our results indicate a significant relation between Bitcoin and the commodity index, as well as between Bitcoin and gold, which is not always symmetric. Furthermore, the results indicate that the relation differs between the short and long runs and across the different quantiles.

The rest of the paper is divided into three sections: section 2 describes the data and methodology; section 3 presents the empirical results; and section 4 concludes.

\section{Data and methodology}

\subsection{Data}

We used daily data prices of Bitcoin and four asset classes (stocks, bonds, commodities, and currencies) for the period between July 17, 2010 and February 2, 2017. The starting date is depicted by the availability of Bitcoin prices that were collected from CoinDesk, which computes and publishes an average of Bitcoin prices from leading trading platforms (Bouri et al. 2017a, b). The data on the four asset classes are from the DataStream of Thomson Reuters, and cover the price index of aggregate S\&P GSCI Commodity (COMMODITY), an ounce of gold (GOLD), MSCI World (MSCI), Pimco investment grade bond (BOND), and the US dollar index (USD). From the statistics of the daily logarithmic level series (Table 1), COMMODITY has the highest average log-price, whereas Bitcoin appears to have both the highest standard deviation. Bitcoin, MSCI and COMMODITY are negatively skewed, meaning that they have longer left tails of the normal distribution, while BOND, GOLD and USD show opposite patterns. All series - except BOND - are platykurtic, indicating that their distributions produce fewer outliers as well as fewer extreme outliers than does a normal 
distribution. The Jarque-Bera test shows that all price series deviate from the normal distribution.

Table 1. Descriptive statistics of daily logarithmic level series

\begin{tabular}{|c|c|c|c|c|c|c|}
\hline & BITCOIN & COMMODITY & GOLD & MSCI & BOND & USD \\
\hline Mean & 3.913 & 8.270 & 7.219 & 7.301 & 2.368 & 4.445 \\
\hline Maximum & 7.045 & 8.661 & 7.549 & 7.502 & 2.489 & 4.637 \\
\hline Minimum & -2.996 & 7.529 & 6.958 & 6.979 & 2.284 & 4.289 \\
\hline Std. Dev & 2.666 & 0.324 & 0.142 & 0.148 & 0.037 & 0.094 \\
\hline Skewness & -0.892 & -0.860 & 0.402 & -0.383 & 0.822 & 0.513 \\
\hline Kurtosis & 2.765 & 2.106 & 2.013 & 1.708 & 3.893 & 1.788 \\
\hline Jarque-Bera & $\begin{array}{c}230.525^{* * *} \\
{[0.000]}\end{array}$ & $\begin{array}{c}267.992^{* * *} \\
{[0.000]}\end{array}$ & $\begin{array}{c}115.503^{* * *} \\
{[0.000]}\end{array}$ & $\begin{array}{c}160.680^{* * *} \\
{[0.000]}\end{array}$ & $\begin{array}{c}249.580^{* * *} \\
{[0.000]}\end{array}$ & $\begin{array}{c}179.602^{* * *} \\
{[0.000]}\end{array}$ \\
\hline
\end{tabular}

Note: $* * *$ indicates rejection of the null hypothesis of normality at the $1 \%$ level.

\subsection{Methodology}

This section presents the methodological approach we used to capture the nonlinear and asymmetric impact of commodity and gold prices on Bitcoin prices. In particular, we employed three nonlinear models, namely the NARDL model (Shin et al., 2011) and the QARDL model (Cho et al., 2015). We further extended the NARDL model to a quantile framework, leading to a new richer model we call the quantile nonlinear ARDL model and denote as QNARDL. Indeed, the NARDL model is employed to disentangle the hidden cointegration introduced by Granger and Yoon (2002). The data is said to have hidden cointegration if its respective positive and negative components are cointegrated, while conventional cointegration tests such as the Engle-Granger (1987) and Johansen (1988) cointegration tests fail to detect cointegrating relationships between level data. In addition, the NARDL model has the advantage of testing cointegration between data series with different orders of integration, in that it allows combining $\mathrm{I}(0)$ and $\mathrm{I}(1)$ data. Furthermore, it provides a nice framework to test for the long- and short-run transmission of commodity and gold prices to Bitcoin prices. However, the NARDL model imposes an exogenous zero threshold, and hence tests the impact of shocks of different signs - positive versus negative - but of the same magnitude/size. The QARDL model is employed to test for a second type of asymmetry known as distributional asymmetry, both in the long and short run according the position of the dependent variable within its own distribution. It is now well documented that asymmetries observed in the relationships between data series are caused by the complex 
systems and sudden events hitting financial and macroeconomic data, as well as their determinants.

The NARDL model accounting for long- and short-run asymmetries for our data is written as: $\triangle B \operatorname{ITCOIN}_{t}=$

$c+\rho_{\text {BITCOIN }}$ ITCOIN $_{t-1}+\rho_{M S C I}^{+} M_{\text {SCCI }}^{+}+\rho_{M S C I}^{-} M_{t-1} C I_{t-1}^{-}+\rho_{B O N D}^{+} B O N D_{t-1}^{+}+$ $\rho_{B O N D}^{-} B O N D_{t-1}^{-}+\rho_{X}^{+} X_{t-1}^{+}+\rho_{X}^{-} X_{t-1}^{-}+\rho_{U S D}^{+} U S D_{t-1}^{+}+\rho_{U S D}^{-} U S D_{t-1}^{-}+$ $\sum_{i=1}^{p-1} \theta_{\text {BITCOIN }} \Delta B$ ITCOIN $_{t-i}+\sum_{i=0}^{q-1}\left(\beta_{M S C I, i}^{+} \Delta M S C I_{t-i}^{+}+\beta_{M S C I, i}^{-} \Delta M S C I_{t-i}^{-}\right)+$ $\sum_{i=0}^{q-1}\left(\beta_{B O N D, i}^{+} \Delta B O N D_{t-i}^{+}+\beta_{B O N D, i}^{-} \Delta B O N D_{t-i}^{-}\right)+\sum_{i=0}^{q-1}\left(\beta_{X, i}^{+} \Delta X_{t-i}^{+}+\beta_{X, i}^{-} \Delta X_{t-i}^{-}\right)+$ $\sum_{i=0}^{q-1}\left(\beta_{U S D, i}^{+} \Delta U S D_{t-i}^{+}+\beta_{U S D, i}^{-} \Delta U S D_{t-i}^{-}\right)+\varepsilon_{t}$

where X refers to commodity or gold. The representation of the NARDL in Eq. 1 is the most flexible one as it accommodates long-run and short-run asymmetries for all the considered determinants of Bitcoin returns. The model uses the decomposition of the explanatory variables $M S C I_{t}, B O N D_{t}, X_{t}$ and $U S D_{t}$ into their respective positive and negative partial sums. The positive partial sums are computed as $\operatorname{MSCI}_{t}^{+}=\sum_{j=1}^{t} \Delta M S C I_{j}^{+}=\sum_{j=1}^{t} \max \left(\Delta M S C I_{j}, 0\right)$, $B O N D_{t}^{+}=\sum_{j=1}^{t} \Delta B O N D_{j}^{+}=\sum_{j=1}^{t} \max \left(\triangle B O N D_{j}, 0\right), X_{t}^{+}=\sum_{j=1}^{t} \Delta X_{j}^{+}=\sum_{j=1}^{t} \max \left(\Delta X_{j}, 0\right)$ and $U S D_{t}^{+}=\sum_{j=1}^{t} \Delta U S D_{j}^{+}=\sum_{j=1}^{t} \max \left(\Delta U S D_{j}, 0\right)$, whereas negative partial sums are computed as $M S C I_{t}^{-}=\sum_{j=1}^{t} \Delta M S C I_{j}^{-}=\sum_{j=1}^{t} \min \left(\Delta M S C I_{j}, 0\right)$, $B O N D_{t}^{-}=\sum_{j=1}^{t} \Delta B O N D_{j}^{-}=\sum_{j=1}^{t} \min \left(\triangle B O N D_{j}, 0\right), \quad X_{t}^{-}=\sum_{j=1}^{t} \Delta X_{j}^{-}=\sum_{j=1}^{t} \min \left(\Delta X_{j}, 0\right)$ and $U S D_{t}^{-}=\sum_{j=1}^{t} \Delta U S D_{j}^{-}=\sum_{j=1}^{t} \min \left(\Delta U S D_{j}, 0\right)$. The NARDL is stable if $\rho_{\text {BITCOIN }}$ is significantly negative. The estimation procedure starts by estimating the NARDL model in Eq. (1) and using the Wald test to test for long- and short-run asymmetries ex-post. In fact, the long-run symmetry of each of the considered determinants was tested using the Wald test of the respective null hypotheses $\rho_{M S C I}^{+}=\rho_{M S C I}^{-}, \rho_{B O N D}^{+}=\rho_{B O N D}^{-}, \rho_{X}^{+}=\rho_{X}^{-}, \quad \rho_{U S D}^{+}=\rho_{U S D}^{-}$, while the short-run symmetries were tested using the Wald test of the respective null hypotheses $\sum_{i=0}^{q-1} \beta_{M S C I, i}^{+}=\sum_{i=0}^{q-1} \beta_{M S C I, i}^{-}, \sum_{i=0}^{q-1} \beta_{B O N D, i}^{+}=\sum_{i=0}^{q-1} \beta_{B O N D, i}^{-}, \sum_{i=0}^{q-1} \beta_{X, i}^{+}=\sum_{i=0}^{q-1} \beta_{X, i}^{-}$ and $\sum_{i=0}^{q-1} \beta_{U S D, i}^{+}=\sum_{i=0}^{q-1} \beta_{U S D, i}^{-}$.

Afterwards, we estimated the QARDL model using the same lag length orders selected for the NARDL model. The QARDL model for our data is given by: 


$$
\begin{aligned}
& Q_{\Delta B I T C O I N}=\alpha(\tau)+\rho(\tau)\left(B I T C O I N-\beta_{M S C}(\tau) M S C_{l} I_{-1}-\beta_{B O N D}(\tau) B O N D_{-1}-\beta_{X}(\tau) X_{t-1}-\beta_{U S D}(\tau) U S D_{-1}\right) \\
& +\sum_{i=1}^{p} \varphi_{i}(\tau) \Delta B I T C O I_{t-1}+\sum_{i=0}^{q 1} \omega_{l}(\tau) \Delta M S C_{-i}+\sum_{i=0}^{q 2} \theta_{i}(\tau) \Delta B O N D_{-i}+\sum_{i=0}^{q 3} \theta_{i}(\tau) \Delta X_{t-i}+\sum_{i=0}^{q 4} \theta_{i}(\tau) \Delta U S D_{-i}+\varepsilon_{t}(\tau)
\end{aligned}
$$

where $\mathrm{X}$ refers to commodity or gold. The symmetry of the speed of adjustment parameter was tested using the Wald test of the null $\rho(0.05)=\rho(0.10)=\rho(0.20)=\rho(0.30)=$ $\rho(0.40)=\rho(0.50)=\rho(0.60)=\rho(0.70)=\rho(0.80)=\rho(0.90)=\rho(0.95) . \quad$ A $\quad$ similar testing procedure was employed to test the symmetry of the long-run and short-run transmissions of the considered explanatory variables to Bitcoin returns.

Combining Eq. (1) and Eq. (2) into the same model gives a more general model that allows testing for distributional asymmetry while accounting for hidden cointegration, short-run and long-run asymmetries. The face resulting QNARDL model is written as:

$$
\begin{aligned}
& \Delta B \operatorname{BTCOIN}_{t}=c+\rho_{\text {BITCOIN }}(\tau) \text { BITCOIN }_{t-1}+\rho_{M S C I}^{+}(\tau) \text { MSCI I }_{t-1}^{+}+\rho_{M S C I}^{-}(\tau) M S C I_{t-1}^{-}+ \\
& \rho_{B O N D}^{+}(\tau) B O N D_{t-1}^{+}+\rho_{B O N D}^{-}(\tau) B O N D_{t-1}^{-}+\rho_{X}^{+}(\tau) X_{t-1}^{+}+\rho_{X}^{-}(\tau) X_{t-1}^{-}+\rho_{U S D}^{+}(\tau) U S D_{t-1}^{+}+ \\
& \rho_{U S D}^{-}(\tau) U S D_{t-1}^{-}+\sum_{i=1}^{p} \theta_{B I T C O I N, i}(\tau) \Delta B I T C O I N_{t-i}+\sum_{i=0}^{q_{1}}\left(\beta_{M S C I, i}^{+}(\tau) \Delta M S C I_{t-i}^{+}+\right. \\
& \left.\beta_{M S C I, i}^{-}(\tau) \Delta M S C I_{t-i}^{-}\right)+\sum_{i=0}^{q_{2}}\left(\beta_{B O N D, i}^{+}(\tau) \Delta B O N D_{t-i}^{+}+\beta_{B O N D, i}^{-}(\tau) \Delta B O N D_{t-i}^{-}\right)+ \\
& \sum_{i=0}^{q_{3}}\left(\beta_{X, i}^{+}(\tau) \Delta X_{t-i}^{+}+\beta_{X, i}^{-}(\tau) \Delta X_{t-i}^{-}\right)+\sum_{i=0}^{q_{4}}\left(\beta_{U S D, i}^{+}(\tau) \Delta U S D_{t-i}^{+}+\beta_{U S D, i}^{-}(\tau) \Delta U S D_{t-i}^{-}\right)+\varepsilon_{t}(\tau)
\end{aligned}
$$

The above model, in Eq. (3), is able to model the following three types of asymmetries:

(i) Reaction asymmetry is measured by the long-run parameters within each quantile. For example, the symmetric long-run impact of MSCI on Bitcoin was tested using the Wald test of the null $\gamma_{M S C I}^{+}(\tau)=\gamma_{M S C I}^{-}(\tau)$ where $\gamma_{M S C I}^{+}(\tau)=-\frac{\rho_{M S C I}^{+}(\tau)}{\rho_{B I T C O I N}(\tau)}$ and $\gamma_{M S C I}^{-}(\tau)=-\frac{\rho_{\overline{M S C I}}^{-}(\tau)}{\rho_{\text {BITCOIN }}(\tau)}$ are the long-run positive and negative parameters.

(ii) Short-run adjustment asymmetry is measured by the short-run parameters within each quantile. For example, the short-run symmetric impact of MSCI on BITCOIN was tested using the Wald test of the null $\beta_{M S C I, i}^{+}(\tau)=\beta_{M S C I, i}^{-}(\tau)$ for $i=$ $0,1, \ldots, q_{1}$.

(iii) Locational asymmetry was captured by the differences between the long- and short-run parameters estimated at the various considered quantiles of the Bitcoin variable. 


\section{Results}

In order to determine the best-suited NARDL specification for our data, we estimated the general NARDL model in Eq. (1) and then tested for long-run and short-run asymmetries using the corresponding Wald tests. The empirical results of the Wald test for long-run and short-run asymmetries for models involving respectively commodity and gold are reported in Table 2. The results indicate that in the model containing COMMODITY as the main explanatory variable, the Wald tests for long-run asymmetries show that MSCI, COMMODITY and USD have a symmetric long-run impact on BITCOIN prices, while BOND has an asymmetric long-run influence on BITCOIN prices. In the short run, all variables i.e. MSCI, BOND, COMMODITY and USD have a symmetric impact on BITCOIN prices. In addition, in the model containing GOLD as the main explanatory variable, MSCI and USD have a long-run asymmetric impact on BITCOIN prices, while BOND and GOLD show a symmetric long-run impact on BITCOIN prices. In the short run, MSCI, BOND, GOLD and USD have a symmetric influence on BITCOIN prices.

Table 2. Results of the long- and short-run Wald tests

\begin{tabular}{|c|c|c|}
\hline & NARDL model involving & NARDL model involving \\
& COMMODITY & GOLD \\
\hline$W L R_{M S C I}$ & 0.887 & $4.153^{* *}$ \\
& {$[0.346]$} & {$[0.042]$} \\
\hline$W L R_{B O N D}$ & $5.701^{* * *}$ & 0.062 \\
& {$[0.003]$} & {$[0.803]$} \\
\hline$W L R_{X}$ & 0.253 & 2.023 \\
& {$[0.615]$} & {$[0.155]$} \\
\hline$W L R_{U S D}$ & 2.242 & $2.247^{*}$ \\
& {$[0.134]$} & {$[0.134]$} \\
\hline$W S R_{M S C I}$ & 1.786 & 1.190 \\
& {$[0.181]$} & {$[0.275]$} \\
\hline$W S R_{B O N D}$ & 1.017 & 0.284 \\
& {$[0.313]$} & {$[0.594]$} \\
\hline$W S R_{X}$ & 0.357 & 0.486 \\
& {$[0.550]$} & {$[0.486]$} \\
\hline$W S R_{U S D}$ & 0.186 & 0.080 \\
& {$[0.666]$} & {$[0.777]$} \\
\hline
\end{tabular}

Notes: Orders of the NARDL models were selected using a general-to-specific approach, by fixing $p_{\max }=$ $q_{\max }=12$. The SIC criterion selected the followings lag-length orders $\mathrm{p}=11, \mathrm{q} 1=0, \mathrm{q} 2=1, \mathrm{q} 3=0, \mathrm{q} 4=0$ for the model including commodity, and $\mathrm{p}=11, \mathrm{q} 1=0, \mathrm{q} 2=0, \mathrm{q} 3=5, \mathrm{q} 4=0$ for model including gold. The $\mathrm{p}$ values are between square brackets. $* * *, * *$ and $*$ indicate rejection of the null hypothesis at the respective significance levels of $1 \%, 5 \%$ and $10 \%$. 
Based on the above analysis, we estimated the following NARDL models for the commodity and gold prices:

$\Delta \operatorname{BITCOIN}_{t}=c+\rho_{\text {BITCOIN }}$ BITCOIN $_{t-1}+\rho_{M S C I} \operatorname{MSCI}_{t-1}+\rho_{B O N D}^{+}$BOND $_{t-1}^{+}+$

$\rho_{B O N D}^{-}$BOND $_{t-1}^{-}+\rho_{\text {COMMODITY }}$ COMMODITY $_{t-1}+\rho_{U S D} U S D_{t-1}+$

$\sum_{i=1}^{p-1} \theta_{\text {BITCOIN }} \Delta$ BITCOIN $_{t-i}+\sum_{i=0}^{q-1} \beta_{M S C I, i} \Delta M_{\text {SCI }}$ I $-i+\sum_{i=0}^{q-1} \beta_{B O N D, i} \Delta B O N D_{t-i}+$

$\sum_{i=0}^{q-1} \beta_{\text {CоммодIтY }, i} \Delta$ COMMODITY $Y_{t-i}+\sum_{i=0}^{q-1} \beta_{U S D, i} \Delta U S D_{t-i}+\varepsilon_{t}$

$\triangle \operatorname{BITCOIN}_{t}=$

$c+\rho_{\text {BITCOIN }}$ BITCOIN $_{t-1}+\rho_{M S C I}^{+}$MSCI I $_{t-1}^{+}+\rho_{M S C I}^{-} M_{S C I}^{-}+\rho_{\text {BOND }} B O N D_{t-1}+$

$\rho_{G O L D} G O L D_{t-1}+\rho_{u s d}^{+} U S D_{t-1}^{+}+\rho_{U S D}^{-} U S D_{t-1}^{-}+\sum_{i=1}^{p-1} \theta_{\text {BITCOIN } \Delta \text { BITCOIN }_{t-i}+}$

$\sum_{i=0}^{q-1} \beta_{M S C I, i} \Delta M S C I_{t-i}+\sum_{i=0}^{q-1} \beta_{B O N D, i} \Delta B O N D_{t-i}+\sum_{i=0}^{q-1} \beta_{C O M M O D I T Y, i} \Delta C O M M O D I T Y_{t-i}+$

$\sum_{i=0}^{q-1} \beta_{U S D, i} \Delta U S D_{t-i}+\varepsilon_{t}$

The estimation results of Eq. (4) and Eq. (5) are reported in Table 3.

Table 3. Estimation results of the NARDL model

\begin{tabular}{|c|c|c|c|}
\hline & COMMODITY & & GOLD \\
\hline Constant & -0.003 & Constant & 0.066 \\
& $(0.381)$ & & $(0.209)$ \\
\hline lbitcoin $_{t-1}$ & $-0.011^{* * *}$ & lbitcoin $_{t-1}$ & $-0.012^{* * *}$ \\
& $(0.003)$ & & $(0.003)$ \\
\hline lmsci $_{t-1}$ & $0.077^{* * *}$ & lmsci $_{t-1}^{+}$ & $0.092^{* *}$ \\
& $(0.028)$ & & $(0.038)$ \\
\hline lbond $_{t-1}^{+}$ & 0.020 & lmsci $_{t-1}^{-}$ & $0.088^{* *}$ \\
& $(0.058)$ & & $(0.036)$ \\
\hline lbond $_{t-1}^{-}$ & -0.039 & lbond $_{t-1}$ & -0.014 \\
& $(0.057)$ & & $(0.065)$ \\
\hline lcommodity $_{t-1}$ & 0.007 & lgold $_{t-1}$ & -0.008 \\
& $(0.017)$ & & $(0.024)$ \\
\hline lusd $_{t-1}$ & $-0.140^{* *}$ & lusd $_{t-1}^{+}$ & $-0.183^{* * *}$ \\
& $(0.067)$ & & $(0.060)$ \\
\hline bitcoin $_{t-3}$ & $0.086^{* * *}$ & lusd $_{t-1}^{-}$ & $-0.211^{* * *}$ \\
& $(0.024)$ & & $(0.074)$ \\
\hline bitcoin $_{t-4}$ & $0.061^{* *}$ & bitcoin $_{t-3}$ & $0.097^{* * *}$ \\
& $(0.024)$ & & $(0.024)$ \\
\hline bitcoin $_{t-5}$ & $-0.049^{* *}$ & bitcoin $_{t-4}$ & $0.073^{* * *}$ \\
& $(0.024)$ & & $(0.024)$ \\
\hline bitcoin $_{t-9}$ & $0.079^{* * *}$ & $\Delta$ bitcoin $_{t-5}$ & $-0.042^{*}$ \\
& $(0.024)$ & & $(0.024)$ \\
\hline bitcoin $_{t-10}$ & $0.048^{* *}$ & bitcoin $_{t-9}$ & $0.075^{* * *}$ \\
& $(0.024)$ & & $(0.024)$ \\
\hline${\text { b } \text { bond }_{t-1}}$ & $0.884^{* *}$ & bitcoin $_{t-10}$ & $0.043^{*}$ \\
& $(0.433)$ & & $(0.024)$ \\
\hline
\end{tabular}




\begin{tabular}{|c|c|c|c|}
\hline & & $\Delta$ gold $_{t-1}$ & $\begin{array}{c}0.477 * * * \\
(0.152)\end{array}$ \\
\hline & & $\Delta$ gold $_{t-3}$ & $\begin{array}{c}-0.354 * * \\
(0.153)\end{array}$ \\
\hline & & $\Delta$ gold $_{t-4}$ & $\begin{array}{c}-0.469 * * * \\
(0.153)\end{array}$ \\
\hline & & $\Delta$ gold $_{t-5}$ & $\begin{array}{c}-0.338 * * \\
(0.153)\end{array}$ \\
\hline$L_{M S C I}$ & $\begin{array}{c}7.070 * * * \\
(2.538)\end{array}$ & $L_{M S C I^{+}}$ & $\begin{array}{c}7.735 * * * \\
(2.737)\end{array}$ \\
\hline$L_{B O N D^{+}}$ & $\begin{array}{c}1.880 \\
(5.389)\end{array}$ & $L_{M S C I^{-}}$ & $\begin{array}{c}7.382 * * \\
(2.834)\end{array}$ \\
\hline$L_{B O N D^{-}}$ & $\begin{array}{c}-3.559 \\
(5.050)\end{array}$ & $L_{B O N D}$ & $\begin{array}{l}-1.194 \\
(5.352)\end{array}$ \\
\hline$L_{\text {COMMODITY }}$ & $\begin{array}{c}0.669 \\
(1.501)\end{array}$ & $L_{G O L D}$ & $\begin{array}{c}-0.674 \\
52.008^{\circ}\end{array}$ \\
\hline$L_{U S D}$ & $\begin{array}{c}-12.908 * * \\
(5.655)\end{array}$ & $L_{U S D^{+}}$ & $\begin{array}{c}-15.370 * * * \\
(3.998)\end{array}$ \\
\hline & & $L_{U S D^{-}}$ & $\begin{array}{c}-17.755^{* * *} * \\
(5.824)\end{array}$ \\
\hline
\end{tabular}

Notes: This table reports the estimation results of NARDL for models including commodity and gold respectively. Only significant short-run coefficients are reported. $* * *, * *$ and indicate significance at the $1 \%, 5 \%$ and $10 \%$ significance levels, respectively.

The empirical results in Table 3 show that the loading parameter of lbitcoin $_{t-1}$ is negative and highly significant in both models. The latter result indicates that the specified NARDL model both for COMMODITY and GOLD is stable.

The results for the model involving COMMODITY as the explanatory variable could be summarized as follows. In the long run, MSCI has a highly significant symmetric positive influence on Bitcoin prices, while USD has a significant symmetric negative long-run influence on Bitcoin prices in the long run. However, BOND and COMMODITY do not exert a significant influence on Bitcoin prices in the long run. In particular, while insignificant, the influence of bond price increases is positive, whereas that of decreases is negative. Turning to the short-run linkages between Bitcoin prices and their considered determinants, the results show that past variations in Bitcoin prices significantly determine their own current variations. The latter cumulative influence is positive, meaning that increases of past variations in Bitcoin prices lead current variations in Bitcoin prices to move up and vice versa. In addition, the one-period lagged variations in bond prices have a positive and significant impact on current variations in Bitcoin prices, whereas in the short run, MSCI, COMMODITY and USD do not exhibit any influence on Bitcoin prices. In particular, the evidence on the positive relation between Bitcoin and bond prices suggests a potential safe 
haven role for Bitcoin in the short run, although the bond market leads the Bitcoin market in the short term. This finding contradicts that of Bouoiyour and Selmi (2015) but is in line with the findings of some prior studies that used different models (e.g., Bouri et al. 2017b,c).

Regarding the model containing GOLD as the main explanatory variable, the results in Table 3 indicate that MSCI has a highly significant asymmetric effect on Bitcoin in the long-run. Indeed, both increases and decreases of MSCI lead Bitcoin prices to decrease, with increases having a greater influence. Similarly, both increases and decreases in USD lead Bitcoin prices to increase, with a greater impact of decreases. In the short run, past variations in Bitcoin prices have a positive cumulative impact on current variations in Bitcoin prices, whereas past variations in gold prices exhibit a significant negative cumulative impact on current variations in Bitcoin prices. While this evidence on the negative relation between Bitcoin and gold prices seems contradictory to the safe haven evidence shown above, it could be partially explained by the time-varying safe haven property of gold. This result contradicts that of Bouoiyour and Selmi (2015), who found an insignificant relation between Bitcoin and gold markets. In contrast, MSCI, BOND and USD do not determine the Bitcoin price in the short run.

We further investigated the distributional asymmetries in the long- and short-run influences of MSCI, COMMODITY (GOLD), BOND and USD on Bitcoin prices, by estimating the model in Eq. (2). Figures 1 and 2 depict the estimated parameters across the quantiles, along with their respective confidence bounds, for models containing respectively COMMODITY and GOLD.

For the model including COMMODITY, the ECM parameter is significant with the negative required sign at the lower quantiles only, while the long-run cointegrating parameter is positively significant at the tail quantiles for MSCI and is insignificant at all quantiles for BOND, COMMODITY and USD, meaning that MSCI exerts a distributional long-run asymmetric effect on Bitcoin prices, while BOND, COMMODITY and USD do not exhibit any influence on Bitcoin prices in the long run. This finding is more nuanced than that reported by Bouoiyour and Selmi (2015), who show a significant relation between Bitcoin and (Chinese) equity prices.

In the short run, past variations in Bitcoin prices have a significant positive effect on their current own variations at the medium to higher quantiles only. USD influences current variations in Bitcoin prices at the extreme high quantile only. Although concentrated in extreme high quantiles, this evidence on the close relation between Bitcoin and the USD points to the role of Bitcoin as a currency (Polasik et al. 2015). In contrast, MSCI, BOND, 
and COMMODITY do not influence Bitcoin prices in the short run. This finding contradicts that of Ciaian et al. (2016), who indicate that US equities and oil prices have a small impact on the Bitcoin price in the short term. This is probably due to the advanced ARDL models that we employed in this study, which allowed us to uncover the first evidence on such a nuanced relation across the different quantiles.

As for the model containing gold, the analysis reflected in the graphs in Fig. 2 indicates that the ECM parameter is significantly negative at the low, and some medium quantiles. The long-run cointegrating parameter is significantly positive at the low and medium quantiles for MSCI, and at the low quantiles for BOND, GOLD and USD.

Current variations in Bitcoin prices are influenced by their own past variations only at the medium to high quantiles. Nevertheless, MSCI, BOND, GOLD and USD do not impact current variations in Bitcoin prices in the short run.

Fig. 3 depicts the estimated QNARDL coefficients across quantiles for the model including COMMODITY. The graphs show that the lagged Bitcoin price coefficient is significantly negative, as required by the cointegration condition at the low to medium quantiles. In addition, increases in bond prices have a positively significant impact on Bitcoin prices in the long run at the low quantiles, while COMMODITY influences Bitcoin prices in the long run positively at the low quantiles and negatively at the high quantiles. In contrast, the MSCI index and decreases in BOND prices do not impact Bitcoin prices in the long run.

In the short run, variations in Bitcoin prices influence their own current variations positively at the high quantiles. The latter significant positive influence is observed at lags four, nine and ten. For the same horizon, MSCI, BOND, COMMODITY and USD do not exhibit any impact on Bitcoin prices in the short run.

Fig. 4 shows the estimated paths of the QNARDL coefficients in the model including GOLD. The speed of adjustment parameter is significantly negative at the low to medium quantiles. Moreover, increases and decreases in MSCI lead Bitcoin prices to move up. Clearly the magnitude of the impact of MSCI increases is higher than that of decreases. BOND, however, impacts Bitcoin prices significantly negatively in the long run at the medium quantiles. Additionally, increases in USD lead Bitcoin prices to move down, while GOLD and decreases in USD do not influence Bitcoin prices at all in the long run. This negative sensitivity of Bitcoin to the USD is often observed in the financial media and empirical studies between commodities (mainly oil and gold) and the USD, suggesting additional similarities between Bitcoin and the commodity markets. 
In the short run, past variations in Bitcoin prices impact their own current variations in a significant positive manner at the medium to high quantiles at different lags, namely lags four, nine, then and eleven. Past variations in GOLD, namely at lags one, three and four, exhibit a significant influence on contemporaneous variations in Bitcoin prices. In contrast, MSCI and USD are not significant determinants of Bitcoin prices in the short run. 
Figure 1. Estimated long- and cumulative short-run parameters of QARDL model containing commodity

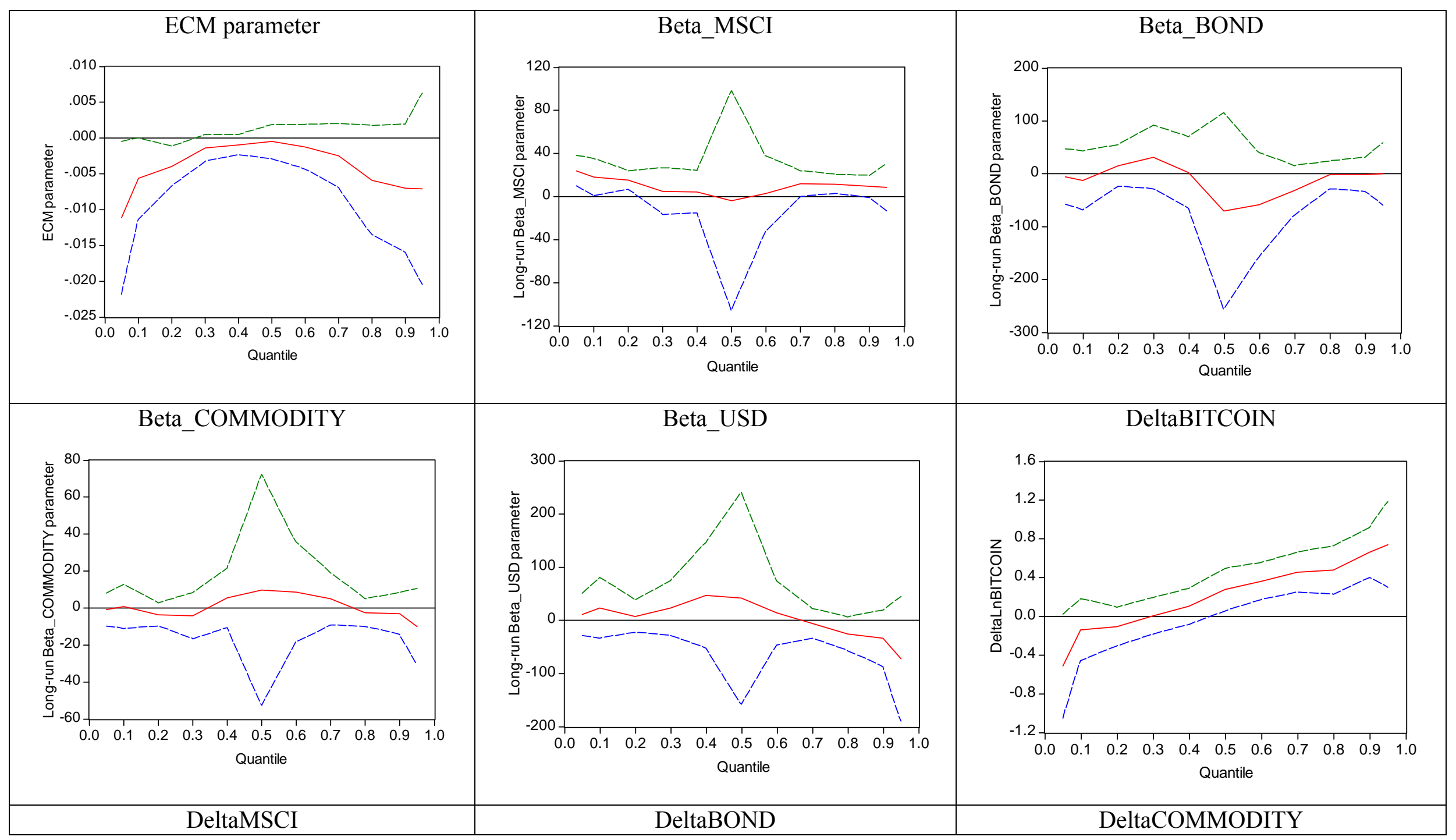




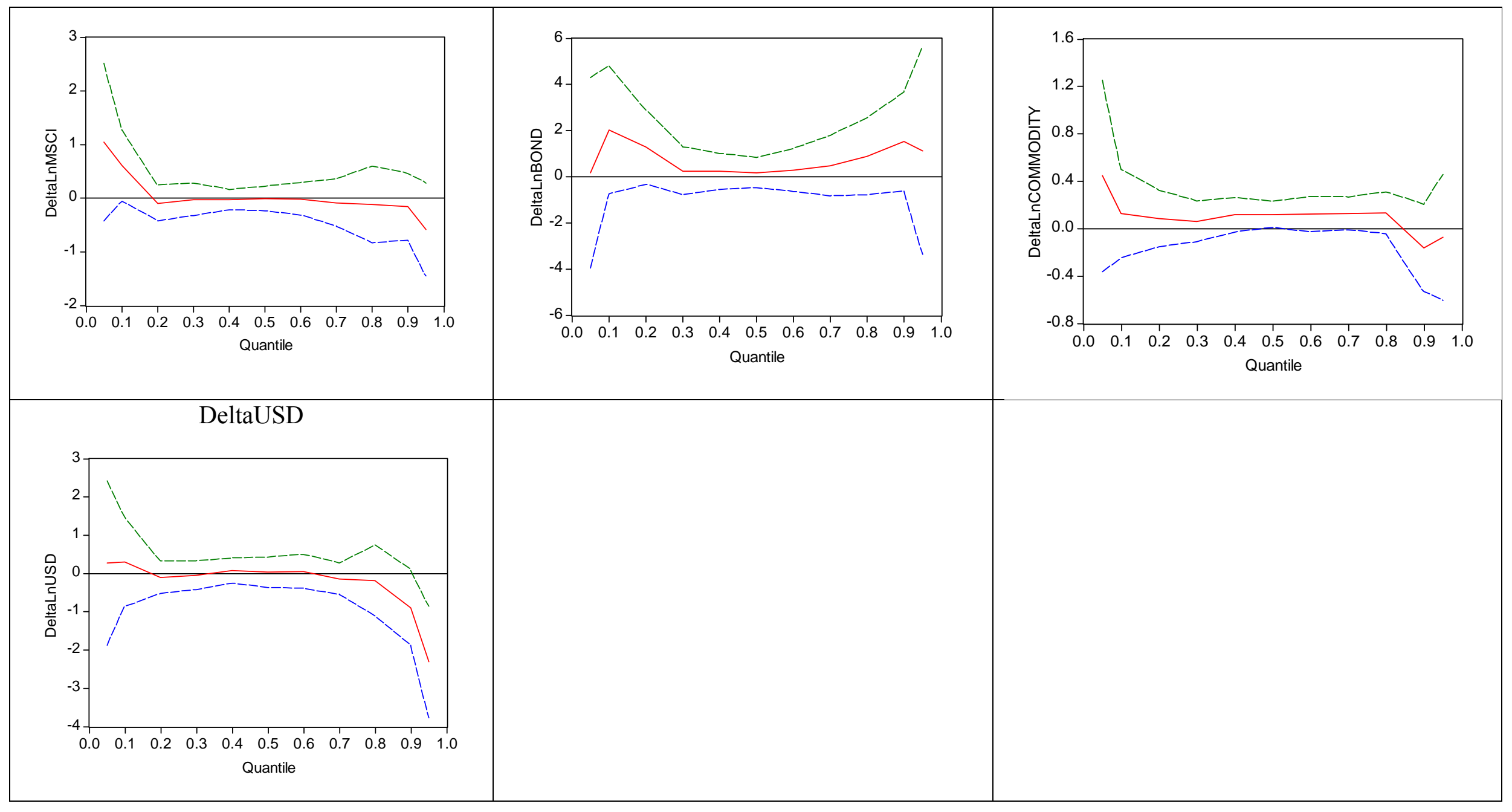


Figure 2. Estimated long- and cumulative short-run parameters of QARDL model containing gold

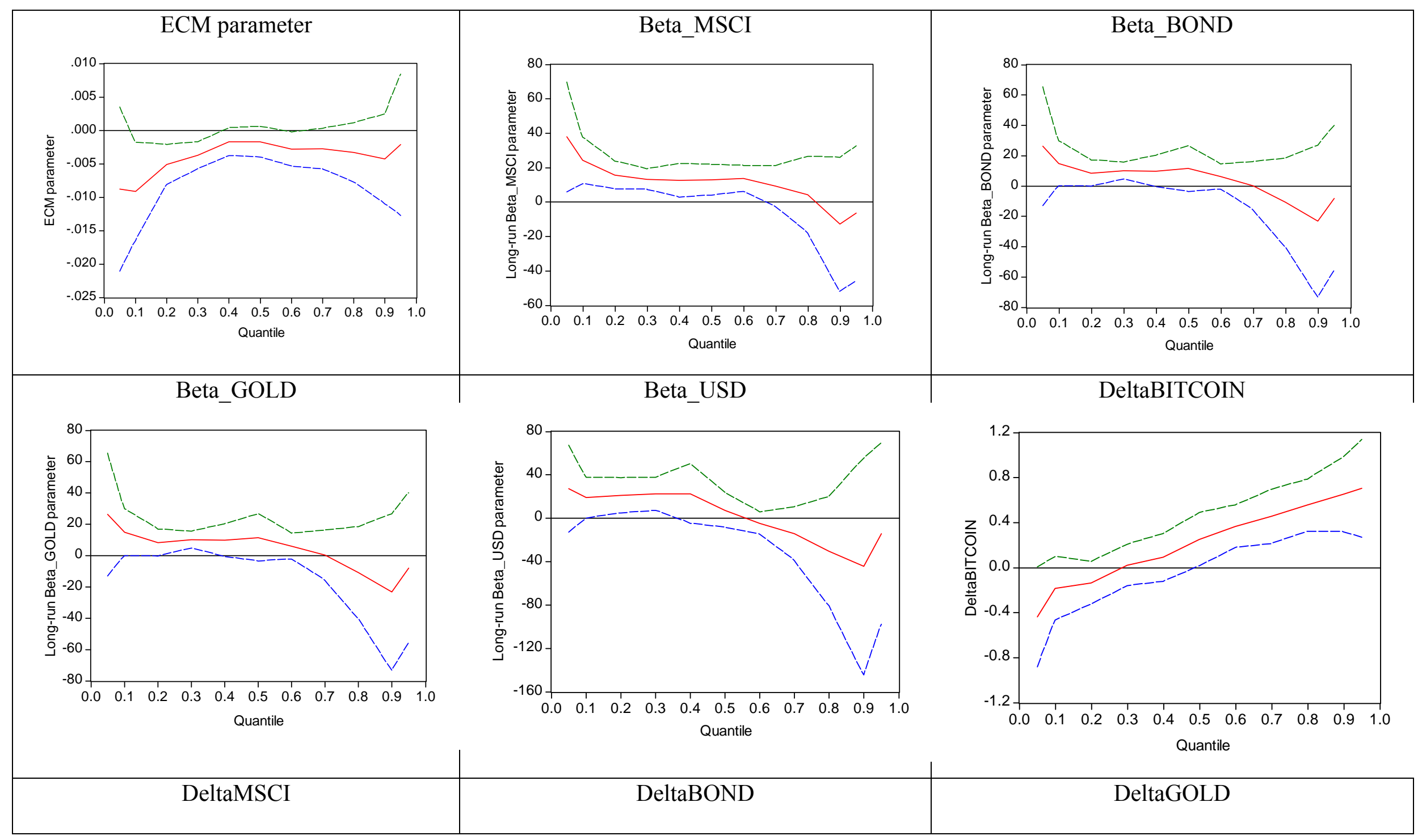




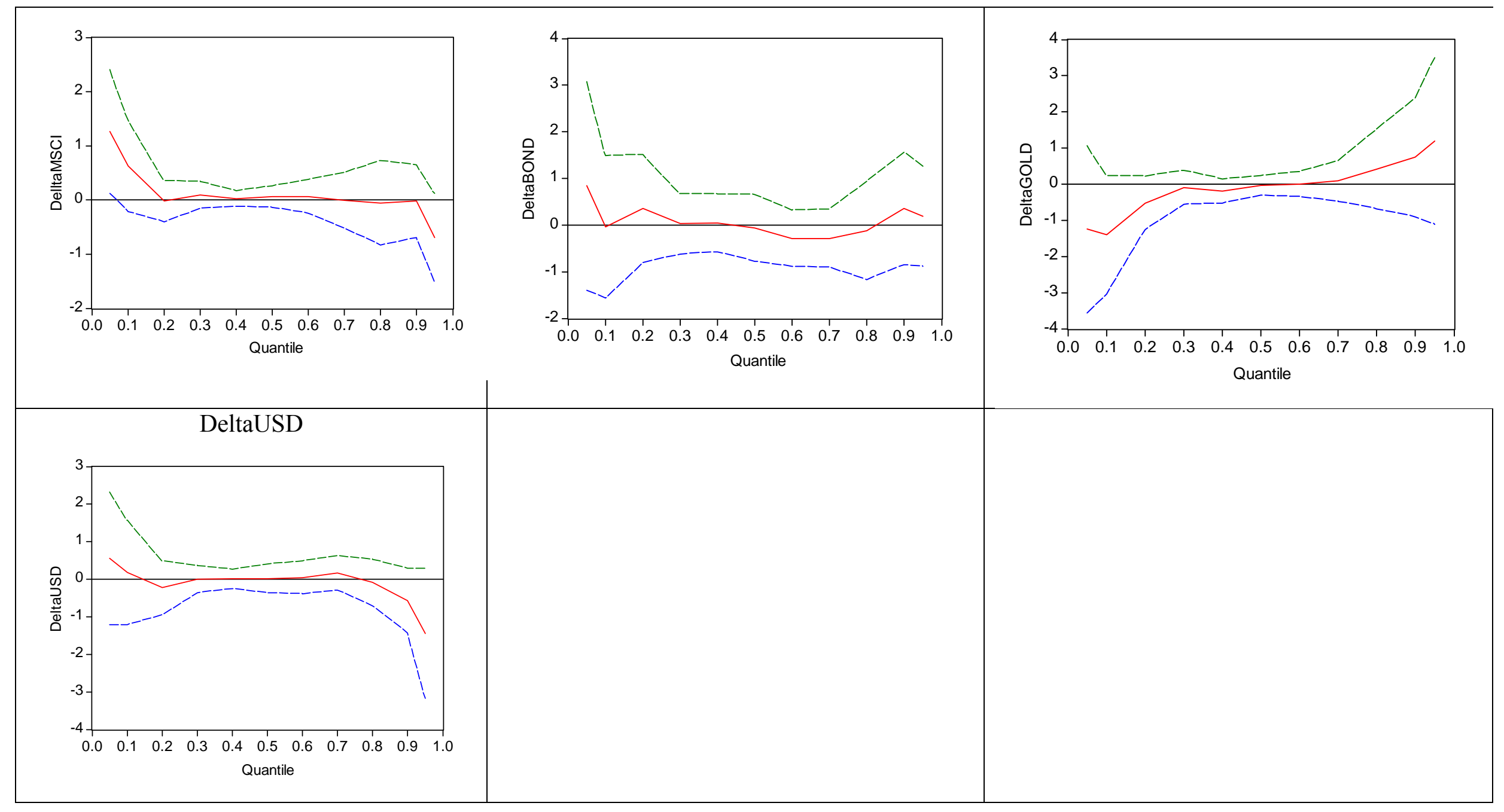


To check for the distributional asymmetry of the QARDL parameters, we carried out the Wald test of the null of parameter constancy across all the considered quantiles, namely 0.05 , $0.1,0.2, \ldots, 0.9,0.95$.

The results in Table 4 indicate that the speed of adjustment parameter shows nonlinear behavior in the model containing COMMODITY. Similarly, in the short-run past, Bitcoin price variations impact their own current variations in an asymmetric manner, basically at lags 2, 4, 9, 10 and 11, where the null of parameter constancy is highly rejected. In addition, current, one-period lagged variations in bond prices and current contemporaneous variations in USD are transmitted to current variations in Bitcoin prices in an asymmetric manner, as the null of parameter constancy across quantiles is also rejected.

In the model containing GOLD, the long-run cointegrating relationship between MSCI and Bitcoin prices is asymmetric, as was shown by the Wald test. Past variations in Bitcoin prices asymmetrically influence their own contemporaneous variations in the short run at some lags, such as at lags 2, 4, 5, and 7-11. More importantly, GOLD influences Bitcoin prices in an asymmetric manner in the short run, both contemporaneously and at different lags.

Table 4. Wald test for asymmetry

\begin{tabular}{|c|c|c|}
\hline & Model 1: COMMODITY & Model 2: GOLD \\
\hline$\rho$ & $1.760^{*}$ & 1.120 \\
& {$[0.064]$} & {$[0.344]$} \\
\hline$\beta_{M S C I}$ & 0.370 & $2.260^{* *}$ \\
& {$[0.961]$} & {$[0.013]$} \\
\hline$\beta_{B O N D}$ & 0.890 & 1.480 \\
& {$[0.541]$} & {$[0.140]$} \\
\hline$\beta_{X}$ & 0.900 & 0.780 \\
& {$[0.529]$} & {$[0.646]$} \\
\hline$\beta_{U S D}$ & 1.590 & 1.460 \\
& {$[0.105]$} & {$[0.149]$} \\
\hline$\Delta \operatorname{LnBITCOIN}_{t-1}$ & 0.870 & 0.830 \\
& {$[0.563]$} & {$[0.596]$} \\
\hline$\Delta \operatorname{LnBITCOIN}_{t-2}$ & $2.410^{* * *}$ & $1.820^{*}$ \\
& {$[0.001]$} & {$[0.053]$} \\
\hline$\Delta$ LnBITCOIN $_{t-3}$ & 1.530 & 1.210 \\
& {$[0.124]$} & {$[0.282]$} \\
\hline$\Delta \operatorname{LnBITCOIN~}_{t-4}$ & $2.530^{* * *}$ & $3.590^{* * *}$ \\
& {$[0.005]$} & {$[0.000]$} \\
\hline$\Delta \operatorname{LnBITCOIN~}_{t-5}$ & 1.210 & $2.440^{* * *}$ \\
& {$[0.283]$} & {$[0.007]$} \\
\hline$\Delta \operatorname{LnBITCOIN}_{t-6}$ & 1.010 & 1.010 \\
& {$[0.430]$} & {$[0.428]$} \\
\hline$\Delta \operatorname{LnBITCOIN}_{t-7}$ & $1.790^{*}$ & $2.790^{* * *}$ \\
\hline
\end{tabular}




\begin{tabular}{|c|c|c|}
\hline & [0.058] & {$[0.002]$} \\
\hline$\Delta$ LnBITCOIN $_{t-8}$ & $\begin{array}{c}1.590 \\
{[0.104]}\end{array}$ & $\begin{array}{c}2.550 * * * \\
{[0.005]}\end{array}$ \\
\hline$\Delta L n B I T C O I N_{t-9}$ & $\begin{array}{c}6.790 * * * \\
{[0.000]}\end{array}$ & $\begin{array}{c}2.380^{* * *} * \\
{[0.008]}\end{array}$ \\
\hline$\Delta \operatorname{LnBITCOIN} N_{t-10}$ & $\begin{array}{c}4.260 * * * \\
{[0.000]}\end{array}$ & $\begin{array}{l}1.800^{*} \\
{[0.056]}\end{array}$ \\
\hline$\Delta \operatorname{LnBITCOIN}_{t-11}$ & $\begin{array}{c}3.170 * * * \\
{[0.000]}\end{array}$ & $\begin{array}{l}1.940 * * \\
{[0.037]}\end{array}$ \\
\hline$\Delta \operatorname{LnMSCI}_{t}$ & $\begin{array}{c}1.010 \\
{[0.436]}\end{array}$ & $\begin{array}{c}1.090 \\
{[0.366]}\end{array}$ \\
\hline$\triangle L n B O N D_{t}$ & $\begin{array}{c}1.830^{*} \\
{[0.051]}\end{array}$ & $\begin{array}{c}0.660 \\
{[0.761]}\end{array}$ \\
\hline$\Delta \operatorname{LnBOND_{t-1}}$ & $\begin{array}{c}4.540 * * * \\
{[0.000]}\end{array}$ & ---- \\
\hline$\Delta \operatorname{Ln} X_{t}$ & $\begin{array}{c}0.900 \\
{[0.536]}\end{array}$ & $\begin{array}{c}2.990 * * * \\
{[0.001]}\end{array}$ \\
\hline$\Delta \operatorname{Ln} X_{t-1}$ & $\begin{array}{ll}--- \\
\end{array}$ & $\begin{array}{c}2.860 * * * \\
{[0.001]}\end{array}$ \\
\hline$\Delta \operatorname{Ln} X_{t-2}$ & ---- & $\begin{array}{c}1.080 \\
{[0.375]} \\
\end{array}$ \\
\hline$\Delta \operatorname{Ln} X_{t-3}$ & $\begin{array}{ll}--- \\
\end{array}$ & $\begin{array}{c}3.570 * * * \\
{[0.000]}\end{array}$ \\
\hline$\Delta \operatorname{Ln} X_{t-4}$ & ---- & $\begin{array}{c}3.340 * * * \\
{[0.000]}\end{array}$ \\
\hline$\Delta \operatorname{Ln} X_{t-5}$ & ---- & $\begin{array}{c}1.310 \\
{[0.219]}\end{array}$ \\
\hline$\Delta \operatorname{LnUSD_{t}}$ & $\begin{array}{c}12.840 * * * \\
{[0.000]}\end{array}$ & $\begin{array}{c}0.750 \\
{[0.674]}\end{array}$ \\
\hline
\end{tabular}

Notes: The above table reports the results of the Wald test for parameter constancy between quantiles. ${ }^{* *}, * *$ and * indicate rejection of the null hypothesis of parameter constancy across quantiles at the $1 \%, 5 \%$ and $10 \%$ significance levels, respectively. 
Figure 3. Estimated long- and short-run parameters of the QNARDL model containing COMMODITY

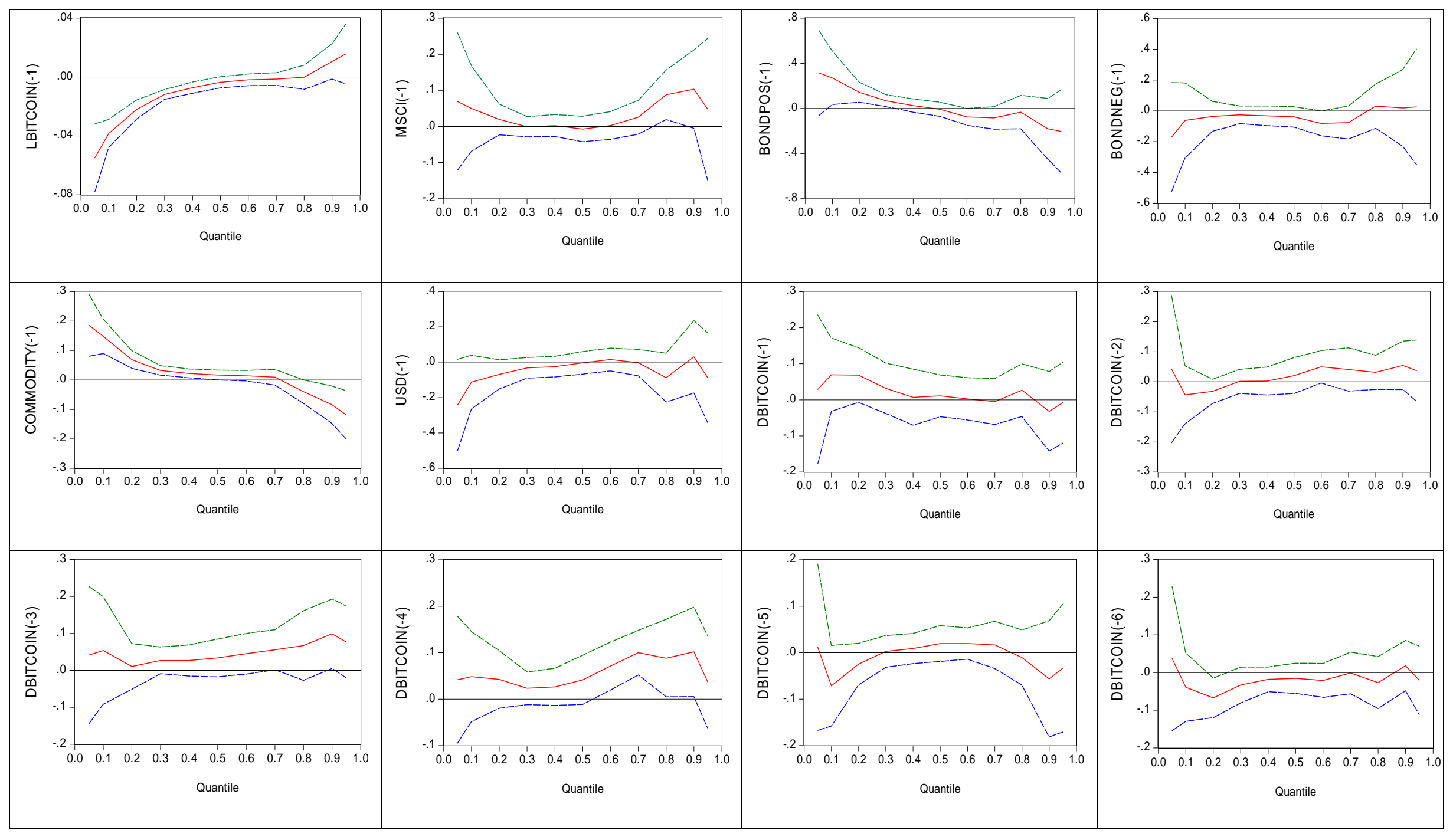




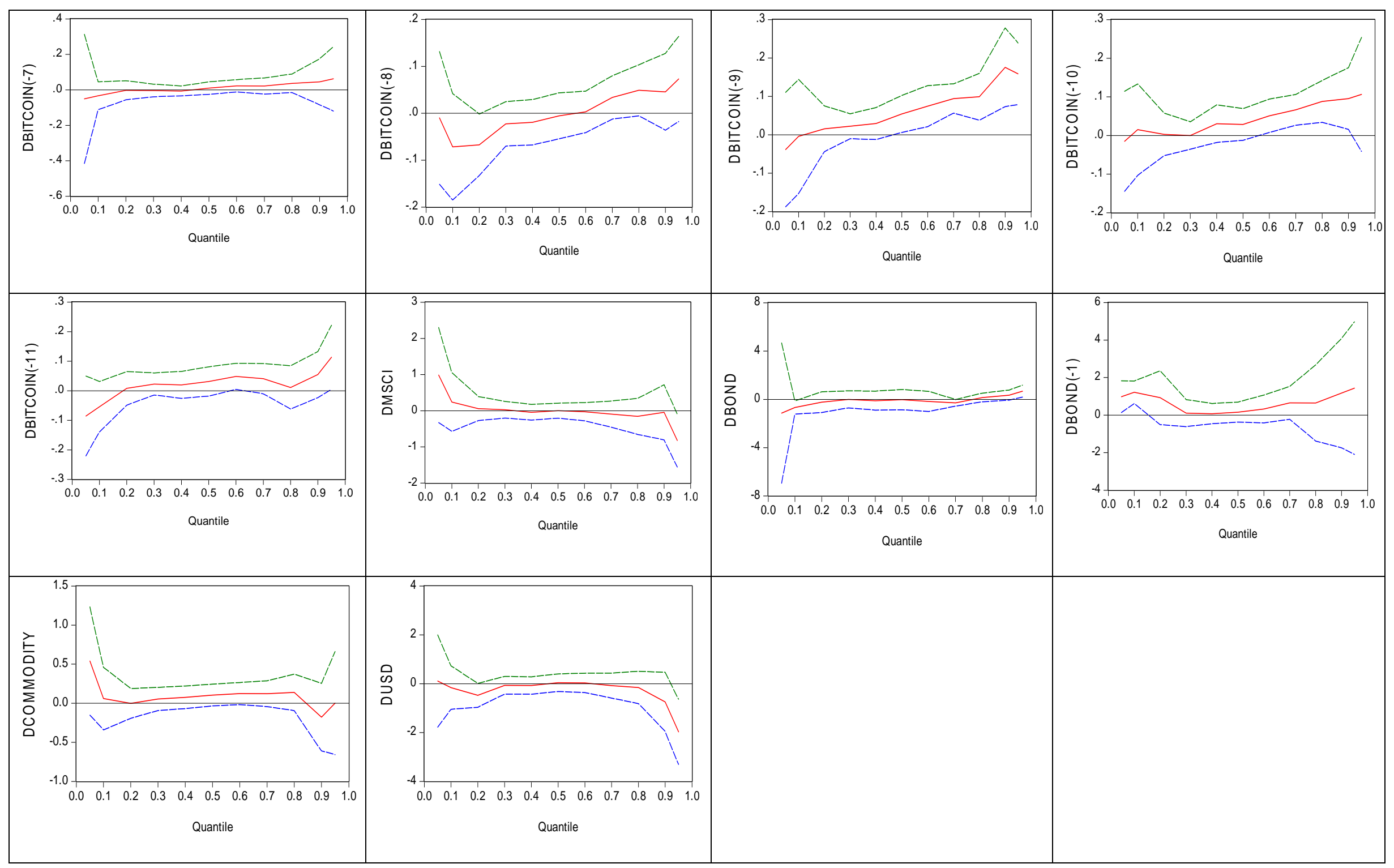


Figure 4. Estimated long- and short-run parameters of the QNARDL model containing GOLD

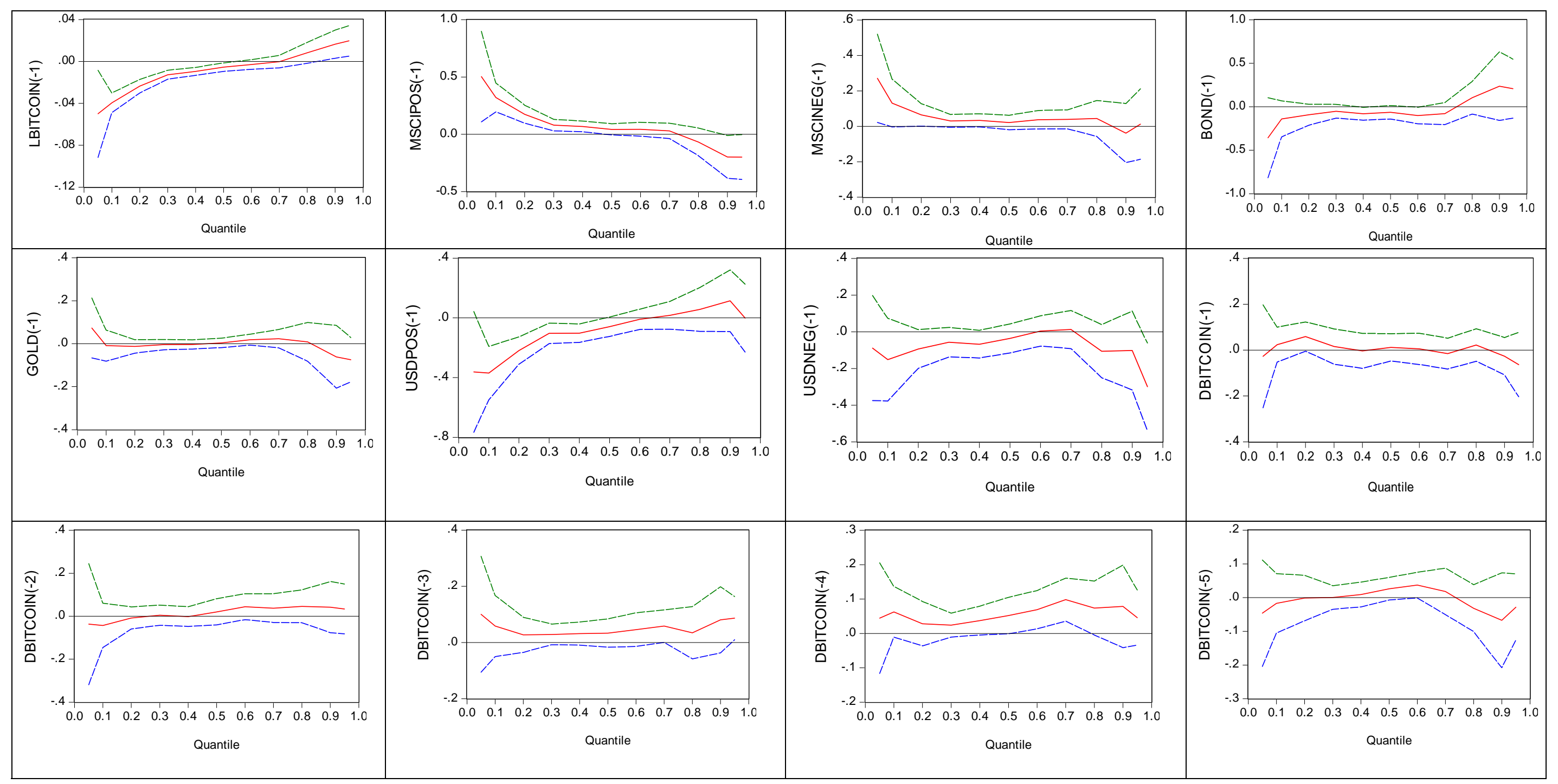




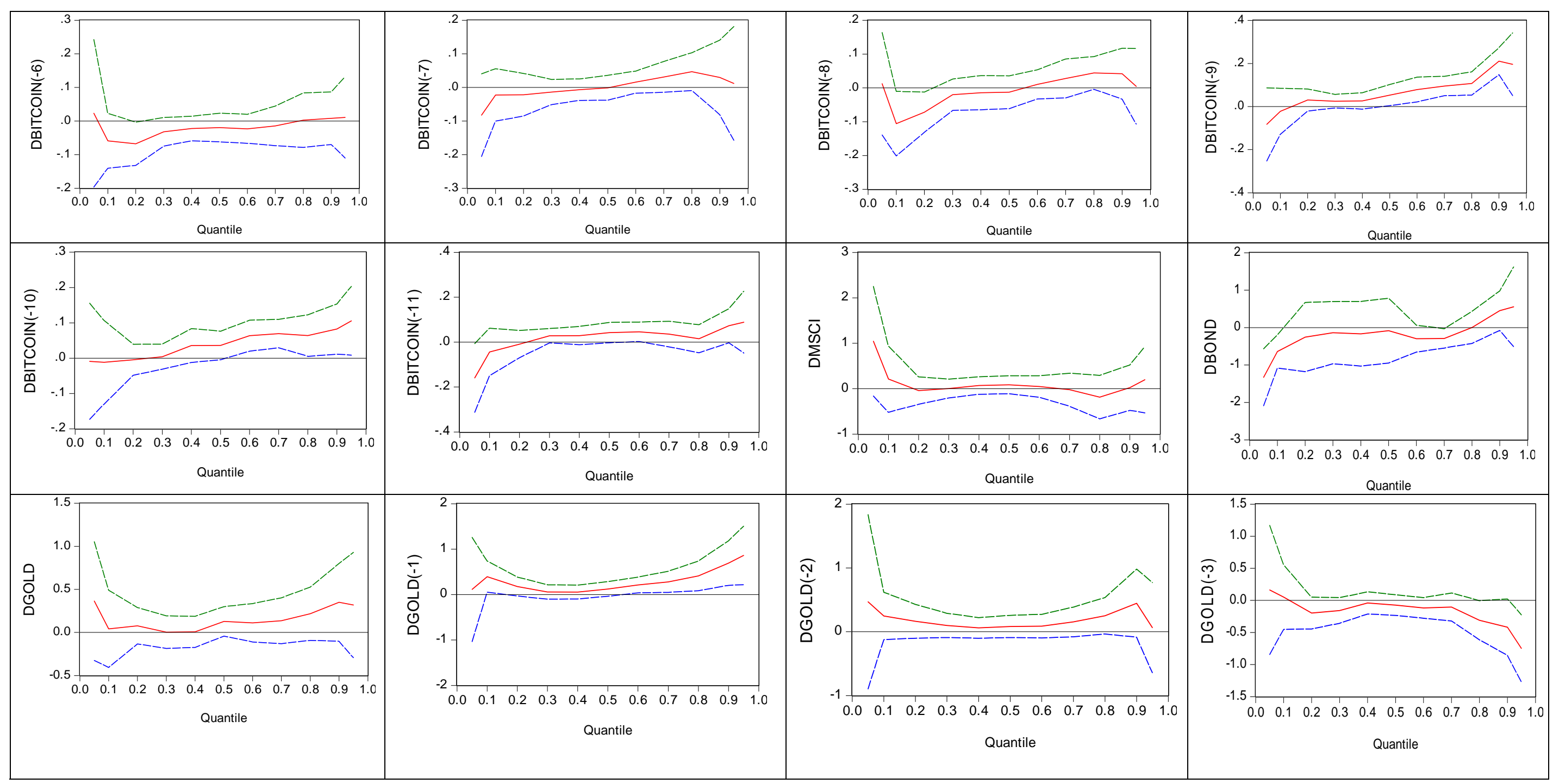




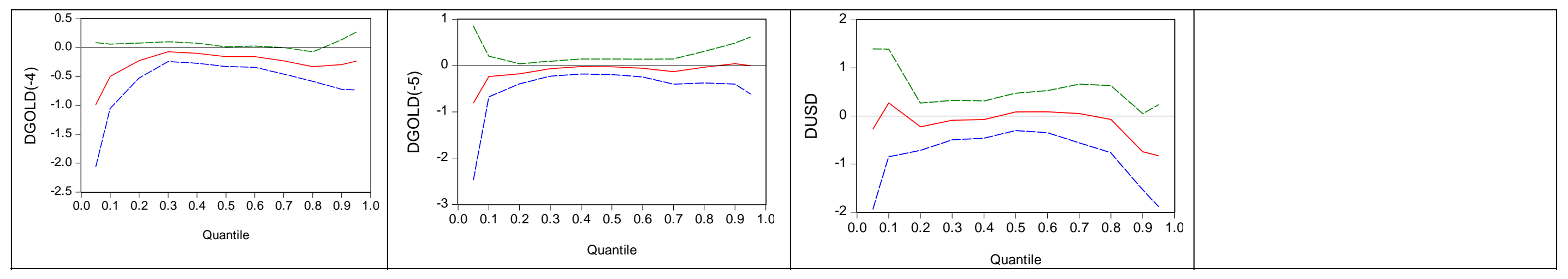


In order to check the distributional asymmetry of the QNARDL model across the considered quantiles, we carried out the Wald test of parameter constancy on each parameter of the two QNARDL models, namely the models including respectively COMMODITY and GOLD. For the first model, including the COMMODITY results in Table 5, below, shows that the ECM parameter is different across quantiles, meaning that the speed of adjustment to the long-run equilibrium path shows asymmetric behavior. Also, increases in bond prices are transmitted to Bitcoin prices asymmetrically across quantiles, while decreases in bond prices pass through Bitcoin prices in a symmetric manner. In addition, COMMODITY has an asymmetric longrun impact on Bitcoin prices. In the short run, Bitcoin returns exert a nine-period lagged asymmetric influence on their current prices, while bond prices and USD pass through Bitcoin prices in an asymmetric manner.

For the second model, including GOLD the Wald test rejects the null of parameter constancy across quantiles for the speed of adjustment parameter, increases and decreases in MSCI, and increases and decreases in USD in the long run. In the short run, variations in Bitcoin prices at lags eight and nine, as well as one-period lagged variations in GOLD are transmitted to current variations in Bitcoin prices in an asymmetric way.

Table 5. Wald test for parameter constancy of QNARDL model

\begin{tabular}{|c|c|c|c|}
\hline & Commodity & & Gold \\
\hline lbitcoin $_{t-1}$ & $75.152^{* * *}$ & lbitcoin $_{t-1}$ & $83.446^{* * *}$ \\
& {$[0.000]$} & & {$[0.000]$} \\
\hline MSCI $_{t-1}$ & 12.857 & MSCI $_{t-1}^{+}$ & $32.953^{* * *}$ \\
& {$[0.232]$} & & {$[0.000]$} \\
\hline BOND $_{t-1}^{+}$ & $27.061^{* * *}$ & MSCI $_{t-1}^{-}$ & 7.936 \\
& {$[0.002]$} & & {$[0.635]$} \\
\hline BOND $_{t-1}^{-}$ & 7.734 & BOND $_{t-1}$ & 12.997 \\
& {$[0.657]$} & & {$[0.223]$} \\
\hline COMMODITY $_{t-1}$ & $43.422^{* * *}$ & GOLD $_{t-1}$ & 11.753 \\
& {$[0.000]$} & & {$[0.302]$} \\
\hline USD $_{t-1}$ & 11.459 & USD $_{t-1}^{+}$ & $29.989^{* * *}$ \\
& {$[0.323]$} & & {$[0.000]$} \\
\hline$\Delta$ LnBITCOIN $_{t-1}$ & 6.410 & USD $_{t-1}^{-}$ & $16.054 *$ \\
& {$[0.780]$} & & {$[0.098]$} \\
\hline LnBITCOIN $_{t-2}$ & 12.607 & $\Delta$ LnBITCOIN $_{t-1}$ & 9.461 \\
& {$[0.247]$} & & {$[0.489]$} \\
\hline$\Delta$ LnBITCOIN $_{t-3}$ & 3.795 & $\Delta$ LnBITCOIN $_{t-2}$ & 4.968 \\
& {$[0.956]$} & & {$[0.893]$} \\
\hline$\Delta$ LnBITCOIN $_{t-4}$ & 13.714 & $\Delta$ LnBITCOIN $_{t-3}$ & 4.084 \\
& {$[0.186]$} & & {$[0.943]$} \\
\hline$\Delta$ LnBITCOIN $_{t-5}$ & 9.275 & $\Delta$ LnBITCOIN $_{t-4}$ & 7.270 \\
& {$[0.506]$} & & {$[0.700]$} \\
\hline
\end{tabular}




\begin{tabular}{|c|c|c|c|}
\hline$\Delta \operatorname{LnBITCOIN} N_{t-6}$ & $\begin{array}{l}10.860 \\
{[0.368]}\end{array}$ & $\Delta \operatorname{LnBITCOIN}_{t-5}$ & $\begin{array}{c}10.577 \\
{[0.391]}\end{array}$ \\
\hline$\Delta \operatorname{LnBITCOIN}_{t-7}$ & $\begin{array}{c}5.942 \\
{[0.820]}\end{array}$ & $\Delta \operatorname{LnBITCOIN}_{t-6}$ & $\begin{array}{c}5.205 \\
{[0.877]}\end{array}$ \\
\hline$\Delta \operatorname{LnBITCOIN}_{t-8}$ & $\begin{array}{c}12.594 \\
{[0.247]}\end{array}$ & $\Delta \operatorname{LnBITCOIN}_{t-7}$ & $\begin{array}{c}7.278 \\
{[0.699]}\end{array}$ \\
\hline$\Delta \operatorname{LnBITCOIN}_{t-9}$ & $\begin{array}{c}20.936^{* *} \\
{[0.021]}\end{array}$ & $\Delta \operatorname{LnBITCOIN} N_{t-8}$ & $\begin{array}{l}16.759 * \\
{[0.080]}\end{array}$ \\
\hline$\Delta \operatorname{LnBITCOIN} N_{t-10}$ & $\begin{array}{l}14.170 \\
{[0.165]}\end{array}$ & $\Delta \operatorname{LnBITCOIN}_{t-9}$ & $\begin{array}{c}38.447 * * * \\
{[0.000]}\end{array}$ \\
\hline${ }_{\Delta L n B I T C O I N}{ }_{t-11}$ & $\begin{array}{l}10.638 \\
{[0.386]}\end{array}$ & $\Delta \operatorname{LnBITCOIN}_{t-10}$ & $\begin{array}{l}15.190 \\
{[0.125]}\end{array}$ \\
\hline$\Delta \operatorname{LnMSCI}_{t}$ & $\begin{array}{c}9.523 \\
{[0.483]}\end{array}$ & $\Delta \operatorname{LnBITCOIN}_{t-11}$ & $\begin{array}{l}12.215 \\
{[0.271]}\end{array}$ \\
\hline$\triangle \operatorname{LnBOND_{t}}$ & $\begin{array}{c}24.238^{* * * *} \\
{[0.007]}\end{array}$ & $\Delta \operatorname{LnMSCI}_{t}$ & $\begin{array}{c}7.088 \\
{[0.717]}\end{array}$ \\
\hline$\triangle L n B O N D_{t-1}$ & $\begin{array}{c}14.792 \\
{[0.140]}\end{array}$ & $\triangle L n B O N D_{t}$ & $\begin{array}{l}17.628^{*} \\
{[0.062]}\end{array}$ \\
\hline$\Delta \operatorname{LnCOMMODITY} Y_{t}$ & $\begin{array}{c}8.324 \\
{[0.597]}\end{array}$ & $\Delta \operatorname{LnGOLD_{t}}$ & $\begin{array}{c}8.107 \\
{[0.618]}\end{array}$ \\
\hline \multirow[t]{6}{*}{$\Delta \operatorname{LnUSD_{t}}$} & $\begin{array}{l}17.213^{*} \\
{[0.070]}\end{array}$ & $\triangle L n G O L D_{t-1}$ & $\begin{array}{l}16.487^{*} \\
{[0.086]}\end{array}$ \\
\hline & & $\Delta L n G O L D_{t-2}$ & $\begin{array}{c}5.150 \\
{[0.881]}\end{array}$ \\
\hline & & $\Delta L n G O L D_{t-3}$ & $\begin{array}{l}14.356 \\
{[0.157]}\end{array}$ \\
\hline & & $\Delta L n G O L D_{t-4}$ & $\begin{array}{c}5.085 \\
{[0.524]}\end{array}$ \\
\hline & & $\Delta L n G O L D_{t-5}$ & $\begin{array}{c}4.441 \\
{[0.925]}\end{array}$ \\
\hline & & $\Delta L n U S D_{t}$ & $\begin{array}{c}9.001 \\
{[0.532]}\end{array}$ \\
\hline
\end{tabular}

Notes: The above table reports the results of the Wald test for parameter constancy between quantiles. ${ }^{* * *}, * *$ and * indicate rejection of the null hypothesis of parameter constancy across quantiles at the $1 \%, 5 \%$ and $10 \%$ significance levels, respectively.

Overall, our results point toward some nuanced relation between the Bitcoin price and the aggregate commodity index (gold prices) that differs not only between the short and long runs but also is asymmetric, non-linear, and quantile dependent. Our main findings are complementary to prior studies that have contributed to the price formation in the Bitcoin market, using standard ARDL models (Ciaian et al. 2016, Li and Wang 2017, Bouoiyour and Selmi 2015). In particular, they contradict with Ciaian et al. (2016), who showed that the Bitcoin price is not driven by global macro-financial development, and with Bouoiyour and Selmi (2015), who found an insignificant relation between Bitcoin and gold markets. However, our findings are in line with those of Li and Wang (2017), who showed that Bitcoin 
is sensitive to macro-economic indicators, although we reveal a broader picture of the specific relation between the Bitcoin and the aggregate commodity index (gold prices), which was found to differ between the short and long runs and across the quantiles. Our analysis therefore highlights the importance of using advanced ARDL models, such as the NARDL, QARDL, and QNARDL to uncover a more complete and nuanced picture of the relation between Bitcoin and asset classes.

Independent of the employed methods, our main findings suggest that the Bitcoin market is not as segmented or isolated as reported by Ji et al. (2017), but instead that it is affected by macro-financial developments, in our case aggregate commodity and gold prices. This finding indicates that Bitcoin and commodity (gold) markets share some common fundamentals, which may reduce the benefits of diversification. Although in line with Bouri et al. $(2017 \mathrm{~b}, \mathrm{c})$, who indicate a very weak correlation between Bitcoin and conventional assets and general commodities, our results contradict with Kristoufek (2015), who argues that the Bitcoin price is not affected by economic and financial variables. We instead show that with appropriate ARDL modelling, which accounts for asymmetry, non-linearity, and quantile dependence, we can indeed predict the Bitcoin price, based on price information from asset classes such as the aggregate commodity index and gold prices. Accordingly, it is recommended that market participants consider relations across the quantiles when making a comparison between Bitcoin and aggregate commodity (gold prices). Furthermore, in exploring the determinants of the Bitcoin price, scholars and financial practitioners should not limit their focus to specific factors of cryptocurrencies (e.g. Bitcoin attractiveness indicators, Ciaian et al. 2016; Blockchain technology, Polasik et al. 2015, Ciaian et al. 2016; anonymity of payment transactions, Ober et al. 2013; use in illegal activities, Böhme et al. 2015; cyber-attacks, Moore and Christin 2013; computer programming enthusiasts, Yelowitz and Wilson 2015), but should also consider macro-financial development variables such as aggregate commodity and gold prices.

\section{Conclusion}

Scholars and practitioners have recently started to examine the relation between Bitcoin and several economic and financial variables. However, very little has been written about Bitcoin and aggregate commodity, in general, and Bitcoin and gold, in particular. Using the advanced ARDL models, we uncovered asymmetric and nonlinear relations between Bitcoin and the aggregate commodity index, as well as between Bitcoin and gold prices, not reported by prior 
studies (Ciaian et al. 2016). We also uncovered more nuanced relations that vary across the quantiles, suggesting that the Bitcoin market is not as segmented or isolated as reported by Ji et al. (2017). Although Bitcoin is part of an alternative economy (Bouri et al. 2017c), its price formation is affected by the aggregate commodity market, in general, and the gold market, in particular. It follows that the Bitcoin market is partially integrated with the global financial system, suggesting some weak diversification benefits from adding Bitcoin to investment portfolios containing other assets. Furthermore, market participants can predict nonlinear and asymmetric movements in the Bitcoin price, based on the price movements of other assets, not on average but in some low and high quantiles.

Armed with such knowledge, Bitcoin users and traders can design trading strategies that involve a large set of financial assets. Thus, there is a possibility of arbitrage, and the Bitcoin market cannot be considered as informationally efficient with respect to aggregate commodity and gold prices. Similarly, for risk management purposes, market participants would have the chance to recognize that aggregate commodity and gold prices represent a portion of the changes in the Bitcoin price. Since Bitcoin does not lodge appeals to intermediaries, it is considered a cheap instrument that policy makers could use to transfer currencies, stocks, commodities, bets and sensitive information.

Although we report noteworthy findings on the relation between Bitcoin and commodity markets, we do not account for the price crash of 2013 (Bouri et al. 2017b) and its potential effect on our analyses. Future research could consider this issue and further focus on the Bitcoin-stock nexus in China, which represents a large part of Bitcoin trading activities.

\section{References}

Baek, C., Elbeck, M. 2015. Bitcoins as an investment or speculative vehicle? A first look. Applied Economics Letters, 22:1, 30-34.

Balcilar, M., Bouri, E., Gupta, R., Roubaud, R. 2017. Can Volume Predict Bitcoin Returns? A Quantiles-based Approach. Economic Modelling, 64, 74-81.

Blau, B. M., 2017. Price dynamics and speculative trading in Bitcoin. Research in International Business and Finance. https://doi.org/10.1016/j.ribaf.2017.07.183.

Bouoiyour, J., Selmi, R. 2015. What does Bitcoin look like? Annals of Economics and Finance, 16(2), 449-492. 
Bouri E., Gupta, R., Tiwari, A., Roubaud, D. 2017a. Does Bitcoin Hedge Global Uncertainty? Evidence from Wavelet-Based Quantile-in-Quantile Regressions. Finance Research Letters. http://dx.doi.org/10.1016/j.frl.2017.02.

Bouri, E., Jalkh, N., Molnár, P., Roubaud, D. 2017b. Bitcoin for Energy Commodities before and after the December 2013 Crash: Diversifier, Hedge or More? Applied Economics, 49(50), 5063-5073.

Bouri, E., Molnár, P., Azzi, G., Roubaud, D., Hagfors L.I. 2017c. On the hedge and safe haven properties of Bitcoin: Is it really more than a diversifier? Finance Research Letters, 20, 192-198.

Brière, M., Oosterlinck, K., Szafarz, A. 2015. Virtual Currency, Tangible Return: Portfolio Diversification with Bitcoins. Journal of Asset Management 16(6) 365-373.

Cheung, A., Roca, E., Su, J.J. 2015. Cryptocurrency bubbles: an application of the PhillipsShi-Yu (2013) methodology on Mt. Gox bitcoin prices. Applied Economics, 47(23), 23482358.

Ciaian, P., Rajcaniova, M., D. Kancs, D. 2016. The economics of Bitcoin price formation. Applied Economics, 48(19), 1799-1815.

Dyhrberg, A. H. 2016a. Bitcoin, gold and the dollar-A GARCH volatility analysis. Finance Research Letters, 16, 85-92.

Dyhrberg, A. H. 2016b. Hedging Capabilities of Bitcoin. Is it the Virtual Gold? Finance Research Letters, 16, 139-144.

Fry, J. and Cheah, E.T., 2016. Negative bubbles and shocks in cryptocurrency markets. International Review of Financial Analysis, 47, 343-352.

Ji, Q., Bouri, E., Gupta, R., Roubaud, D. 2017. Network Causality Structures among Bitcoin and other Financial Assets: A Directed Acyclic Graph Approach. University of Pretoria, Department of Economics Working Paper (No. 201729).

Kristoufek, L., 2015. What are the main drivers of the Bitcoin price? Evidence from wavelet coherence analysis. PloS one, 10(4), e0123923. 
Li, X., Wang, C.A. 2017. The technology and economic determinants of cryptocurrency exchange rates: The case of Bitcoin. Decision Support Systems, 95, 49-60.

Lo, S., Wang, C. J. 2014. Bitcoin as Money? Current Policy Perspectives, No. 14-4, Federal Reserve Bank of Boston, Boston, MA.

Luther, W.J., Salter, A.W. 2017. Bitcoin and the Bailout. The Quarterly Review of Economics and Finance. https://doi.org/10.1016/j.qref.2017.01.009.

Moore, T. and Christin, N. 2013, April. Beware the middleman: Empirical analysis of Bitcoin-exchange risk. In International Conference on Financial Cryptography and Data Security (pp. 25-33). Springer Berlin Heidelberg.

Nakamoto, S. 2008. Bitcoin: A peer-to-peer electronic cash system.

Polasik, M., Piotrowska, A. I., Wisniewski, T. P., Kotkowski, R., Lightfoot, G. 2015. Price fluctuations and the use of Bitcoin: An empirical inquiry. International Journal of Electronic Commerce, 20(1), 9-49.

Popper, N. 2015. Digital gold: The untold story of Bitcoin. London: Penguin.

Weber, B. 2014. Bitcoin and the legitimacy crisis of money. Cambridge Journal of Economics, 40(1), 17-41.

Yelowitz, A., Wilson, M. 2015. Characteristics of Bitcoin users: an analysis of Google search data. Applied Economics Letters, 22:13, 1030-1036.

Yermack, D., 2013. Is Bitcoin a real currency? An economic appraisal (No. w19747). National Bureau of Economic Research. URL: http://www.nber.org/papers/w19747. 